\title{
Effect of Clinical Pathway on Satisfaction of Patients Undergoing Thyroid surgery
}

\author{
Abdo M. Alhusami ${ }^{1}$, Mimi M. Mekkawy ${ }^{2}$, Abdullah M. Mojelly ${ }^{3}$, Madiha A. Mahmoud ${ }^{4}$. \\ 1. Medical, Surgical Nursing, Faculty of Medicine \& Health Sciences, Thamar University, Yemen. \\ 2. Medical, Surgical Nursing, Faculty of Nursing, Assiut University, Egypt. \\ 3. General surgery, Faculty of Medicine \& Health Sciences, Sanaa University, Yemen. \\ 4. Medical, Surgical Nursing, Faculty of Nursing, Assiut University, Egypt.
}

\begin{abstract}
The patient satisfaction is a health care outcome indicator. The clinical pathway (CP) has evolved as an important tool for enhancing efficient healthcare. The aim of the study was to measure the effect of CP implementation on satisfaction of hospitalized patients undergoing thyroid surgery. The study was conducted at the Al-Thawra Modern General Hospital which is located in Sana'a capital of Yemen. The consecutive patients admitted between June 2014 and October 2014, were enrolled to the conventional care group $(n=30)$ and the consecutive patients admitted between November 2014 and March 2015, were recruited to the CP group $(n=30)$. Pre-operative assessment and Patient's satisfaction questionnaire tools were used for data collection. The results revealed that the patients in the CP group had higher level of satisfaction than the conventional care group (Mean \pm SD: $109.9 \pm 15.4$ versus 83.6 \pm 14.6 ). The results also revealed that there were strong negative correlation between $\mathrm{CP}$ outcomes (length of hospital stay and complications) and patient satisfaction (respectively, $r=-0.514, r=-0.76$ ) Conclusion: The implementation of $\mathrm{CP}$ in the management of patients undergoing thyroid surgery had increased significantly the level of patients' satisfaction regarding admission, patient care, education, length of stay and complications.
\end{abstract}

\section{Key word: Clinical Pathway, Satisfaction \& Thyroid Surgery.}

\section{Introduction}

Thyroid surgery is the most frequent of endocrine surgeries and its rate has tripled over the last three decades. It is also a high-volume that impacts health care services worldwide (Khanzada, et al., 2010 \& Chandrasekhar, et al., 2013).

Nowadays, the challenges in patients' care, in particularly, surgical patients, across different disciplines are adjustments in the care process to reduce variations in clinical practice, improve interdisciplinary cooperation, integrate care, and engineer the efficient use of shrinking resources while maintaining or even increasing quality of outcomes in patient management with patients satisfaction (Ramanujam \& Cheah, 2005). Several methodologies have been advocated. A major organizational strategy is a CP (Barbieri, et al., 2009).

CPs are a methodology for the mutual decision making and organization of care for a well-defined group of patients during a well-defined period. CPs are designed by multidisciplinary cooperation and implemented by organizational and technical measures. As a result, CPs are being increasingly used in health settings and recommended by broader health systems internationally as a form of quality improvement (Graeber, et al., 2007 \& Dubuc, et al., 2013).

Purposes of pathways include: defining standards for expected length of stay (LOS) and for use of specific tests and treatments; giving all team members a plan and specific roles; decreasing nursing and physician documentation burdens; providing a framework for collecting data; and educating and involving patients and families in their care; and provide better care through a mechanism that is able to coordinate clinical processes and to reduce unjustified variations and, ultimately, costs (Hussain, et al., 2017).

Simultaneously, the concept of the patient satisfaction as an outcome measure has gained importance as health care providers move beyond achievable care quality and patient safety targets and start organizing multi-disciplinary methods of care driven by new key performance indicators, such as patient satisfaction (Kash \& Mckahan, 2017).

Many authors tend to have many definitions of patient satisfaction. Iftikhar et al., (2011) indicated that patient satisfaction mostly represents attitudes towards care or aspects of care. While Rama \& Kanagaluru (2011) referred to patient satisfaction as patients' emotions, feelings and their perception of delivered healthcare services. Other authors defined patient satisfaction as a degree of congruency between patient expectations of ideal care and their perceptions of real care received (Al-Abri \& AlBalushi, 2014)

Given this, in Al-Thawra Modern General HospitalYemen, the researchers have developed the first CP for the management of patient undergoing thyroid surgery which incorporates the currently available evidence-based management. Patient satisfaction was 
one of the important indicators of this pathway in which this study was designed.

\section{Significance of the study}

Care for patients undergoing thyroid surgery tends to be appropriate for $\mathrm{CP}$ development because of highvolume, high-cost of the procedure and the predictable course of events that occur during the hospitalization. Patient satisfaction is considered a major indicator of care quality and reflects patients' involvement in decision making. This is the first study in this location to clarify the relationship between patient satisfaction and effectiveness of $\mathrm{CP}$ as quality improvement tool of patients care.

\section{The Aim of the study}

The aim of the study was to measure the effect of $\mathrm{CP}$ implementation on satisfaction of hospitalized patients undergoing thyroid surgery.

\section{Research hypotheses}

Two research hypotheses were formulated to fulfill the aim of the study:

- CP group would exhibit higher level of satisfaction than the conventional care group does.

- There would be relationship between patient satisfaction and favorable CP outcomes (LOS and complications).

\section{Patients \& Method}

1. Research design: Quasi-experimental design was used to this study.

2. Setting: The study was conducted at the General Surgical Department (120 bedded) of Al-Thawra Modern General Hospital which is an 880 bedded multispecialty hospital, which is governed by Ministry of Health \& Population, Republic of Yemen and located in Sana'a capital of Yemen.

3. Sample: Consecutive patients $(n=34)$ scheduled for thyroid surgery, were recruited from June 2014 till October 2014, and these constituted the control group receiving conventional care. Patients $(n=32)$ scheduled for thyroid surgery with the application of the CP were recruited from November 2014 till March 2015, from Al-Thawra Modern General Hospital, in Sana'a, in Yemen. Patients $(n=6)$ with other major operation, aged 18 and younger, or aged 65 and older were excluded. Our analyses were finally based on 60 patients.

\section{Tools of data collection:}

Two data collection tools were utilized to collect the necessary information for the study. The following tools were used:

Tool I: Pre-operative Assessment Tool

This tool was developed by researchers based on reviewing related literatures, tested for validity and was utilized to collect data regarding the demographic data and clinical data. This tool consisted of two parts.

Part one: Demographic data of the study sample this part was developed by researchers to identify the patients' demographic characteristics included 9 questions about age, sex, marital status, residence, occupation, surgery indication, type of operation, date and time of admission and date and time of discharge. This information was provided by combination of patient interview and review of patient's record.

Part two: clinical data of the study sample: this part was developed by researchers to identify patients' clinical information included 9 questions after testing validity about present history, past medical and surgical histories, comorbidities ,smoking history (within one year before surgery). This information was provided by combination of patient interview and review of patient's record.

\section{Tool II: Patient's Satisfaction Questionnaire}

This tool was developed by researchers based on reviewing related literatures including existing satisfaction tools to assess patient's satisfaction about the quality of perioperative care services. It has six domains and 40 items after testing validity and analyzing internal consistency. Hospital admission procedure domain (has 4 items), patient care domain (has 10 items), information provision domain (has13 items), the personal treatment domain (has 5 items) food quality domain (has 3 items) and hospital facilities domain (has 5 items).

All domains are in a 4-point Likert format that goes from strongly satisfied (4) to strongly dissatisfied (1), which helps in obtaining an ordinal measure of the strength of the satisfaction with each item. The total score of the tool ranges from 40 to 160 . The score of $\leq 80=$ dissatisfied, the range score of $>80-120=$ satisfied, and the range score of $>120-160=$ very satisfied.

Cronbach's alpha for the overall tool was 0.96 . Interitem correlation was 0.44 . Item to total correlation ranged from $0.41-0.85$.

\section{Method}

Phase one: "Assessment phase"

During this phase, Patient's socio-demographic data, baseline characteristics and perioperative data were documented and recorded in the tool one. The patients' satisfaction questionnaire (tool two) was administered by an interviewer to both groups at discharge. Furthermore, the clinical outcomes of those patients were evaluated during this phase.

Phase two: "Establishing the clinical pathway" This phase was accomplished by the following steps: Step 1: Selection of an expert panel Expert panel was involved in each step of pathway development. This panel was composed of the 
following: (i) two general surgery professors from faculty of medicine in Sanaa-Yemen; (ii) two professors in faculty of nursing in Assuit-Egypt who are specialized in medical surgical nursing and have extensive clinical and scientific expertise in development of CP; (iii) the supervisor, the head nurse and two staff nurses; (iv) one physiotherapist; (v) one nutritionist; and (vi) the researcher.

\section{Step 2: Literature review}

An extensive literature review was conducted by the researchers to identify all available evidence and studies of pathways used for management of patients undergoing thyroidectomy.

\section{Step 3: Formulation of clinical pathway}

The CP was redrafted twice before the final agreed format was ready. The estimated length of stay for patients undergoing partial thyroidectomy is 2 days, including the day of operation and one postoperative day. The estimated length of stay for patients undergoing total thyroidectomy is 3 days, including the day of operation and two postoperative days.

The CP for patients undergoing partial thyroidectomy formulated into three time-lined phases including: admission (day 1), day of surgery (day 2) and discharge (day 3) and the CP for patients undergoing total thyroidectomy formulated into for time-lined phases including: admission (day 1), day of surgery (day 2), pre-discharge (day 3) and discharge (day4).The established CP consists of five parts:

Part I: Pre-operative evaluation and preparation

This part includes (1) preoperative assessment (such as patient health history and physical examination);( 2) consultations (such as anesthesiologist and medical doctor for medical fitness for operation);(3)diagnostic investigation;(4) preoperative treatments and care (such as blood pressure , hypothyroidism if indicated, skin preparation, and preparing cross-matching blood);(5) pre-medications(such as benzodiazepine at $11 \mathrm{pm}$ and medications prescribed on anaesthesia chart);(6) nutrition(fasting preparation 8 hours before operation); (7) activities and (8)patient and family teaching (such as information about operation and postoperative exercises).

\section{Part II: Post-operative daily interventions}

This part includes the daily nursing and collaborative interventions including (1) assessment ; (2) consultations ; ( 3) diagnostic tests (for total thyroidectomy: serum calcium after 6hours, and calcium iodide after two days) (4) treatments and care ;( 5) medications; (6) nutrition (start oral fluids 6hours after operation); (7) activities (sit up after 6 hours); (8) patient and family education; and (9) discharge planning. The discharge criteria for those undergoing partial thyroidectomy are as follows: 1)no fever;2)no dyspnea or stridor 3) pain controlled with oral analgesics; 4) wound in good condition;5) 24- drainge less than or equal $50 \mathrm{ml}$ with drain removed before discharge;6)oral tolerance of a liquid or semisoft diet. The discharge criteria for those undergoing total thyroidectomy is the same as for patients undergoing partial thyroidectomy but with the addition of:1)absence of clinical signs of tetany; and 2)calcium iodide readings $\geq 4.0 \mathrm{mg} / \mathrm{dl}$ ( $1 \mathrm{mmol} / \mathrm{L})$.

\section{Part III: Expected daily outcomes}

This part includes the patient's daily outcomes such as vital signs stability, pain control, and meeting discharge criteria.

\section{Part IV: Variance tracking record}

This part notes any variations occurring in, or not covered by, the schedule of CP matrix. The solution adopted for each specific case is indicated.

Part V: Protocol for calcium replacement

This protocol is used for treating postoperative hypocalcaemia in patients undergoing total thyroidectomy.

\section{Step 4: Evaluating of content validity}

Content validity of the CP was evaluated and examined by 11 academic and clinical experts. The Content Validity Index (CVI) was used to estimate if the $\mathrm{CP}$ developed would meet all criteria of evaluation. According to the CVI index, a rating of three or four indicates the content is consistent with any criteria. Each criteria was considered met if the level of agreement between reviewers was 0.78 or above.

\section{Step 5: Piloting}

A pilot study was conducted on $10 \%$ of $\mathrm{CP}$ group to evaluate the feasibility of the $\mathrm{CP}$ and to modify the pathway accordingly.

Step 6: Dissemination and implementation strategy

Staff members who, involved in patient care, composed of 6 nurses and 5 physicians involved in patient care were met in separate sessions scheduled over a two weeks period to explain the pathway in brief and oultline the main roles to be played by each one.

Phase Three: "Implementing the clinical pathway"

During this phase, the developed CP was applied to the $\mathrm{CP}$ group for approximately five months started in November 2014. Staff members who, involved in patient care, implemented the established pathway on the $\mathrm{CP}$ group from admission till discharge under researcher's supervision and the $\mathrm{CP}$ group assessment ,meanwhile, was taken and documented as described in phase one.

Phase Four: "Evaluating the clinical pathway outcomes"

The efficacy of the pathway was determined by comparing outcomes for patients of both 
conventional care and $\mathrm{CP}$ groups including patient satisfaction.

\section{Ethical considerations:}

Patients' respect, privacy and information confidentiality were protected using a numbered code on all the questionnaires. No names or identifying information was gathered on the questionnaires. The researchers maintained all questionnaires in a locked and secure file. The data was entered into Statistical Package for Social Sciences statistical software, version 16 , using only the numeric identification code to identify patients. After finishing data analysis, all of the administered questionnaires were destroyed.

\section{Statistical analysis}

The collected data were organized, tabulated and statistically analyzed using the statistical package for social studies (SPSS) version 16. Variables were presented as number and percentage and differences between conventional care group and $\mathrm{CP}$ group were compared using chi square test. For variables with $2 \mathrm{X} 2$ categories where chi square was not suitable for the presence of small observations with expected values less than 5, Fisher exact test was used instead. The level of significance was adopted at $\mathrm{p}<0.05$. The independent $t$ test is used for comparing mean variables between the conventional care group and CP group.

\section{Results}

Table (1): Preoperative assessment of patients undergoing thyroid surgery in conventional and CP groups in relation to patients' demographic data $(n=60)$.

\begin{tabular}{|c|c|c|c|}
\hline \multirow{2}{*}{$\begin{array}{l}\text { Patient's demographic } \\
\text { characteristics }\end{array}$} & $\begin{array}{c}\text { Conventional care group } \\
(\mathbf{n}=\mathbf{3 0})\end{array}$ & $\begin{array}{l}\text { Clinical Pathway group } \\
(n=30)\end{array}$ & \multirow[t]{2}{*}{ P value } \\
\hline & n (\%) & n (\%) & \\
\hline $\begin{aligned} \text { Age (Yr): } & \\
& \cdot 18-<35\end{aligned}$ & $8(26.7)$ & $11(36.7)$ & \multirow[t]{3}{*}{0.676} \\
\hline - $35-<50$ & $16(53.3)$ & $13(43.3)$ & \\
\hline - 50-65 & $6(20)$ & $6(20)$ & \\
\hline - Mean \pm SD & $38.77 \pm 12.01$ & $38.50 \pm 11.8$ & 0.931 \\
\hline $\begin{array}{r}\text { Gender: } \\
\text { - Male }\end{array}$ & $4(13.3)$ & $3(10)$ & \multirow[t]{2}{*}{0.688} \\
\hline - Female & $26(86.7)$ & $27(90)$ & \\
\hline $\begin{array}{c}\text { Marital status: } \\
\text { - Single }\end{array}$ & $2(6.7)$ & $3(10)$ & \multirow[t]{2}{*}{0.640} \\
\hline - Married & $28(93.3)$ & $27(90)$ & \\
\hline $\begin{array}{c}\text { Level of education: } \\
\text { - Illiterate }\end{array}$ & $25(83.3)$ & $24(80)$ & \multirow[t]{5}{*}{0.174} \\
\hline - Read and Write & $0(0)$ & $4(13.3)$ & \\
\hline - Basic Education & $1(3.3)$ & $1(3.3)$ & \\
\hline - $2^{\text {nd }}$ Education & $2(6.7)$ & $1(3.3)$ & \\
\hline - University & $2(6.7)$ & $0(0)$ & \\
\hline $\begin{array}{r}\text { Working status: } \\
\text { - Worker }\end{array}$ & $0(0)$ & $3(10)$ & \multirow[t]{4}{*}{0.158} \\
\hline - Housewife & $25(83.3)$ & $25(83.3)$ & \\
\hline - Employee & $2(6.7)$ & $0(0)$ & \\
\hline - Not working & $3(10)$ & $2(6.7)$ & \\
\hline $\begin{array}{l}\text { Smoking Status: } \\
\text { (Within 1yr before surgery) } \\
\text { - Smoker }\end{array}$ & $1(3.4)$ & $0(0)$ & \multirow[t]{2}{*}{0.306} \\
\hline - Nonsmoker & $29(96.6)$ & $30(100)$ & \\
\hline
\end{tabular}


Table (2): Preoperative clinical data assessment of patients undergoing thyroid surgery in both conventional and clinical pathway groups $(\mathrm{n}=60)$.

\begin{tabular}{|c|c|c|c|}
\hline \multirow[b]{2}{*}{ Clinical data } & Conventional Care group $(n=30)$ & Clinical Pathway group $(n=30)$ & \multirow[t]{2}{*}{$P$ value } \\
\hline & $\mathrm{n}(\%)$ & $\mathrm{n}(\%)$ & \\
\hline $\begin{array}{l}\text { Indication of thyroid surgery: } \\
\text { - Simple goiter }\end{array}$ & $14(46.7)$ & $19(63.3)$ & \multirow[t]{4}{*}{0.135} \\
\hline - Adenomas & $3(10)$ & $1(3.3)$ & \\
\hline - Follicular neoplasia & $1(3.3)$ & $4(13.3)$ & \\
\hline $\begin{array}{c}\text { - Thyroid cancers } \\
\text { - Papillary } \\
\text { - Follicular } \\
\text { - Anaplastic }\end{array}$ & $\begin{array}{c}12(40) \\
11(91.7) \\
0(0) \\
1(8.3)\end{array}$ & $\begin{array}{c}6(20) \\
5(83.3) \\
1(16.7) \\
0(0)\end{array}$ & \\
\hline $\begin{aligned} \text { Type of operation: } \\
\text { - Total thyroidectomy }\end{aligned}$ & $24(80)$ & $21(70)$ & \multirow[t]{2}{*}{0.371} \\
\hline - Hemi thyroidectomy & $6(20)$ & $9(30)$ & \\
\hline $\begin{array}{r}\text { Clinical presentation: } \\
\text { - Neck swelling }\end{array}$ & $30(50)$ & $30(50)$ & ------ \\
\hline - Hoarseness & $4(6.7)$ & $7(11.7)$ & 0.317 \\
\hline - Pain & $6(10)$ & $4(6.7)$ & 0.488 \\
\hline - Dyspnea & $5(8.3)$ & $5(8.3)$ & 1.000 \\
\hline - Thyrotoxic symptoms & $6(10)$ & $3(5)$ & 0.278 \\
\hline - Dysphagia & $4(6.7)$ & $5(8.3)$ & 0.718 \\
\hline - Hypothyroidism & $1(1.7)$ & $0(0)$ & 0.500 \\
\hline $\begin{array}{l}\text { Past medical history: } \\
\text { - Yes }\end{array}$ & $7(23.4)$ & $4(13.4)$ & \multirow[t]{2}{*}{.3170} \\
\hline - No & $23(76.6)$ & $26(86.6)$ & \\
\hline $\begin{array}{c}\text { Surgical history: } \\
\text { - Yes }\end{array}$ & $4(13.4)$ & $3(10)$ & \multirow[t]{2}{*}{0.688} \\
\hline - No & $26(86.6)$ & $27(90)$ & \\
\hline $\begin{array}{c}\text { Co-morbidity: } \\
\text { • Yes }\end{array}$ & $4(13.4)$ & $5(16.6)$ & \multirow[t]{2}{*}{0.718} \\
\hline - No & $26(86.6)$ & $25(83.4)$ & \\
\hline $\begin{array}{c}\text { Types of co-morbidity: } \\
\text { - Hypertension }\end{array}$ & $1(3.3)$ & $2(6.7)$ & 0.554 \\
\hline - Diabetes mellitus & $1(3.3)$ & $2(6.7)$ & 0.554 \\
\hline - Peptic ulcer & $1(3.3)$ & $1(3.3)$ & ------ \\
\hline - Epilepsy & $1(3.3)$ & $0(0)$ & 0.306 \\
\hline - Liver & $1(3.3)$ & $0(0)$ & 0.306 \\
\hline
\end{tabular}

Fisher's Exact test and Pearson Chi-Square test. Significant level $P$ at $\leq 0.05$.

Table (3): Comparison of patients satisfaction mean score between conventional care group and clinical pathway group $(n=60)$.

\begin{tabular}{|l|c|c|c|}
\hline \multirow{2}{*}{\multicolumn{1}{c|}{ Patient satisfaction }} & Conventional care group(n=30) & Clinical pathway group $(\mathbf{n}=\mathbf{3 0})$ & \multirow{2}{*}{ P } \\
\cline { 2 - 4 } & Mean \pm SD & Mean \pm SD & $0.034^{*}$ \\
\hline Admission procedure & $7.4 \pm 5.6$ & $9.55 \pm 1.9$ & $0.009^{* * *}$ \\
\hline Patient care & $21.4 \pm 4.9$ & $33.5 \pm 8.0$ & $0.045^{*-}$ \\
\hline Information provision & $26.2 \pm 8.0$ & $39.5 \pm 12.19$ & 0.976 \\
\hline Personal treatment & $8.4 \pm 3.20$ & $8.45 \pm 3.39$ & 0.748 \\
\hline Food quality & $6.8 \pm 1.30$ & $6.45 \pm 2.16$ & 0.671 \\
\hline Hospital facilities & $13.4 \pm 2.7$ & $12.4 \pm 4.46$ & $0.006^{* * *}$ \\
\hline Total score & $83.6 \pm 14.6$ & $109.9 \pm 15.4$ & \\
\hline
\end{tabular}

Independent t-test was used. Significant level $P \leq 0.05 . *=$ Significant. $* *=$ High significant 


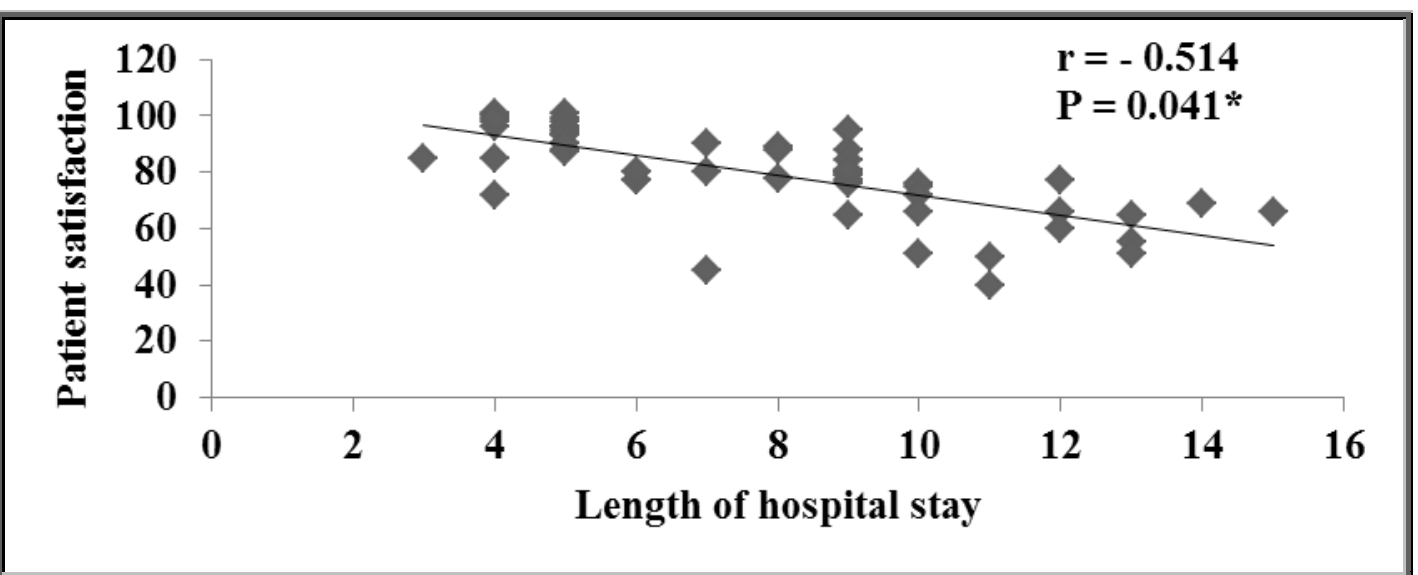

Figure(1): Correlation between length of hospital stay and patients satisfaction.

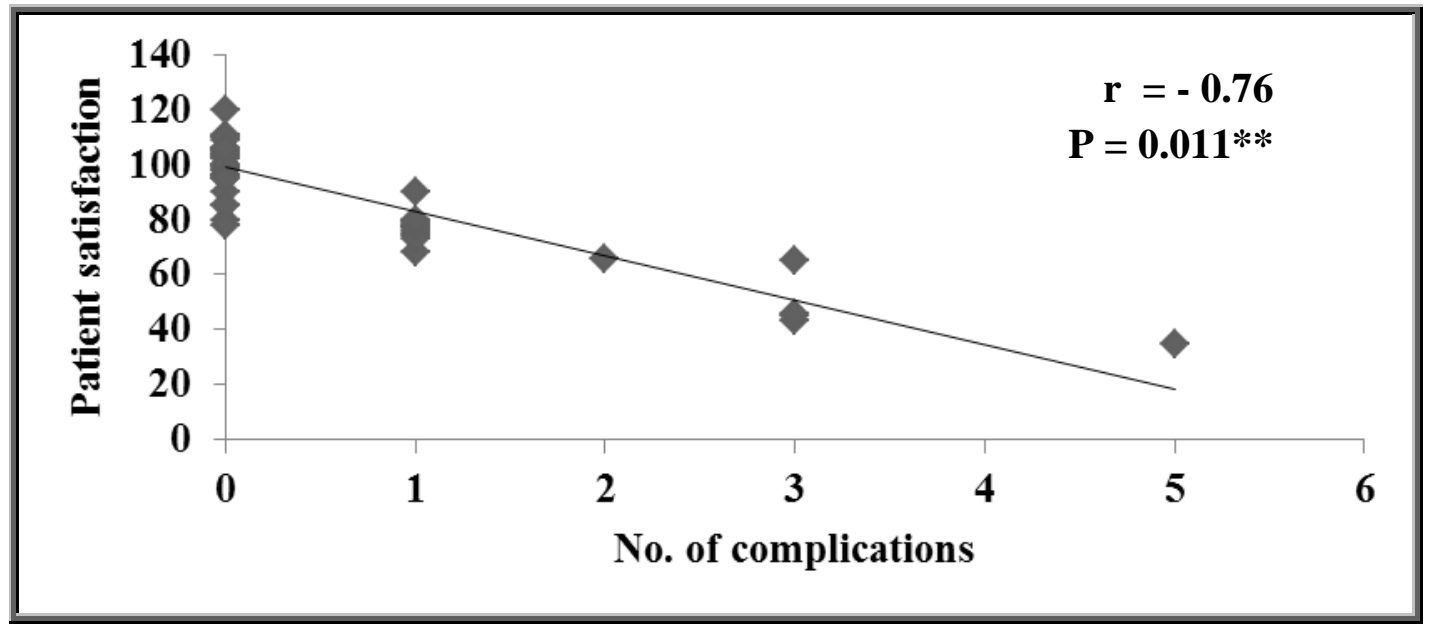

Figure (2): Correlation between complications and patients satisfaction.

\section{Pre-Operative Assessment}

Table (1): Shows that, the distributions of patients' demographic data between the conventional care and $\mathrm{CP}$ groups were comparable and no significant differences were noted in age, gender, marital status, level of education, working status and smoking status $(\mathrm{p}>0.05)$.

Table (2): Shows that, no differences were noted in all preoperative clinical data $(p>0.05)$ between the conventional care and $\mathrm{CP}$ groups.

\section{Patient Satisfaction Assessment}

Table (3): Pretends that, the total satisfaction mean score for conventional care group was 83.6 \pm 14.6 while the total satisfaction mean score for $\mathrm{CP}$ group was $109.9 \pm 15.4$. There was statistically high difference between conventional care group and $\mathrm{CP}$ group in the total mean satisfaction score $(\mathrm{P}<0.001)$.

The Table (3): Also notes that, the patients were more satisfied with admission procedure regarding $\mathrm{CP}$ group compared to conventional care group $(9.55 \pm 1.9$ vs $7.4 \pm 5.6, p<0.05)$. Patients were more satisfied with patient care process and quality regarding $\mathrm{CP}$ group compared to conventional care group $(33.5 \pm 8.0$ vs $21.4 \pm 4.9$, p $<0.001)$. Patients were more satisfied with amount and quality of information provision regarding $\mathrm{CP}$ group compared to conventional care group (39.5 \pm 12.19 vs $26.2 \pm 8.0$, $\mathrm{P}<0.05)$.

The Table (3): Also shows that, the conventional care group and CP group were similarly satisfied with personal treatment $(8.4 \pm 3.20$ vs $8.45 \pm 3.39)$; food quality ( $6.8 \pm 1.30$ vs $6.45 \pm 2.16)$; and no statistically significant difference between conventional care group and $\mathrm{CP}$ group with hospital facilities (13.4 $\pm 2.7 \mathrm{vs} 12.4 \pm 4.46, \mathrm{p}>0.05)$.

The Figures $(1,2)$ : Show that there was strong negative correlation between $\mathrm{CP}$ outcomes (length of hospital stay and complications) and patient satisfaction (respectively, $\mathrm{r}=-0.514, \mathrm{r}=-0.76$ ). 


\section{Discussion}

Thyroid surgery is a routinely common practiced surgery with a low incidence of morbidity, and mortality is rare. It is both a high-volume and medium-cost procedure that impacts health care services and financing (Ramanujam \& Cheah, 2005 \& Berri \& Houar, 2013).

CPs have been identified as an effective tool to guide clinicians in providing evidence-based health care, enhancing uptake of clinical practice guidelines in routine care and are explicit about the sequence, timing and provision of interventions, and have the potential to improve the quality of care by guiding clinical assessments and interventions at the point of care. Further, CPs may lead to improved clinical outcomes and reduced health care costs (Elliott, et al., 2017).

In the Surgery Department at the selected hospital, the researchers developed and implemented a CP in 2014. The objective of the study was to investigate the "outcomes of the CP implementation for patients undergoing thyroidectomy". In this pathway, the researchers collected data on a variety of variables as length of stay, complications and patient satisfaction then the researchers analyzed, reviewed and worked on the finding. As part of this pathway the researchers conducted this study, and it has provided an important in understanding the effect of $\mathrm{CP}$ on patient satisfaction (Ahmed, et al., 2017).

As a baseline for comparison between the conventional care and the $\mathrm{CP}$ groups, both groups had similar access to General Surgical Department care services and there were no any significant differences in patients' demographic and clinical data. Patient satisfaction is a valuable outcome measure because it is a major determinant of the patient willingness to continue taking the care. Patient satisfaction is influenced by many factors, such as treatment regimen, general well-being of the patient, and the quality of communication between the patient and medical care providers (Yang, et al., 2014).

In the current study, the researchers found that there was significant difference between both groups in relation to overall patient satisfaction; in other words, this study confirmed the stated hypothesis that expected higher level of satisfaction in $\mathrm{CP}$ group. These results could be explained by the overall patient's satisfaction regarding ease of overall admission procedure, focused comprehensive care, and thorough continuous education about the operation and care that introduced to $\mathrm{CP}$ group patients and their families throughout the whole perioperative period was in favor of patients with $\mathrm{CP}$. A meta-analysis, conducted by Yang et al., (2014) included six controlled trials that all reported use of a clinical nursing pathway increased patient satisfaction. The study finding were also in line with a study of Soria et al., (2008) that showed the satisfaction was influenced by the overall perception of the process and the additional information and diagrams explaining the process that patients were given.

The patients were more satisfied with admission procedure regarding $\mathrm{CP}$ group compared to conventional care group. This could be explained by schedule appointment times of investigations and operation, and waiting time in admission office. This study finding was in agreement with Hussain (2017) \& Mohammed, et al (2019).

The patients were more satisfied with patient care process and quality regarding $\mathrm{CP}$ group compared to conventional care group. This could reflect adequacy of all aspects of care, cooperation among staff from multiple disciplines and using $\mathrm{CP}$ protocols to help improve practice, coordination and to improve the quality of perioperative treatment by standardizing care. This finding was compatible with study of AlQalah \& Mukhtar (2017).

Patients were more satisfied with amount and quality of information provision regarding $\mathrm{CP}$ group compared to conventional care group. This could reflect thorough education that patients in this group received along perioperative period and on discharge. This finding was in line with Hussain (2017) \& Mohammed, et al., (2019).

In the present study, the results revealed also that there was strong negative correlation between $\mathrm{CP}$ outcomes (length of hospital stay and complications) and patient satisfaction. These results could also explain the higher level of satisfaction in CP group who had shorter length of stay and lesser complication rate. The study data concerning patient's satisfaction came in concordance with other published results of the same interest (Husted, et al., 2008; Aldaqal, et al., 2012, Garcı, et al., 2014 \& Kaye, et al., 2017).

From the former discussion, it is evident that patients who underwent CP for thyroid surgery had significantly increased patients' satisfaction. It could be concluded that application of the $\mathrm{CP}$ was very effective in achieving this positive satisfaction.

\section{Conclusion}

Based on the main results of the present study, it can be concluded that, the implementation of $\mathrm{CP}$ in the management of patient undergoing thyroid surgery had improved significantly the level of patients' satisfaction and patient satisfaction is influenced by many factors, such as ease of overall admission procedure, schedule appointment time of operation, focused comprehensive care, and thorough continuous education that introduced to $\mathrm{CP}$ group 
patients and their families, shorten length of hospital stay and less complications.

\section{Recommendations}

Based on the findings of this study, following recommendations were made:

- The improvement of patient satisfaction suggests that there is significant benefit to apply the $\mathrm{CP}$ to the care of hospitalized patients undergoing thyroid surgery.

- The multidisciplinary team's satisfaction, after implementation of the CP for thyroid surgery should be evaluated.

\section{References}

1. Ahmed, A., Mekkawy, M., \& Mojelly, A., (2017): Outcomes of clinical pathway implementation for patients undergoing thyroidectomy, $1^{\text {st }}$ international conference faculty of nursing, Assuit, Eygpt,30 Jun.2017: 280-291.

2. Al-Abri, R., \& Al-Balushi, A., (2014): Patient satisfaction survey as a tool towards quality improvement. Oman Medical Journal, 29(1), 3 7.

3. Aldaqal, S., Alghamdi, H., Alturki, H., Eldeek, B., \& Kensarah, A., (2012): Determinants of patient satisfaction in the surgical ward at a university hospital in Saudi Arabia. Life Science Journal; 9(1), 277-280.

4. Al-qalah, T., \& Mukhtar, H., (2017): Development and implementation of a clinical pathway for care of cardiac surgical patients. International Journal of Science and Research (IJSR); 6(11), 1626-1631.

5. Barbieri, A., Vanhaecht, K., Van Herck, P., Sermeus, W., Faggiano, F., Marchisio, S., \& Panella, M., (2009): Effects of clinical pathways in the joint replacement: a metaanalysis. BMC Medicine, 7(1), 32.

6. Berri, T., \& Houar, R., (2013): Case report Complications of thyroidectomy for large goiter. Pan African Medical Journal, 16: 138.

7. Chandrasekhar, S., Randolph, G., \& Seidman, M., (2013): Clinical practice guideline: improving voice outcomes after thyroid surgery. Otolaryngology-Head and Neck Surgery. 2013; 148(6): 1-37.

8. Dubuc, N., Bonin, L., Tourigny, A., Mathieu, L., Couturier, Y., Tousignant, M., \& Raîche, M., (2013): Development of integrated care pathways: toward a care management system to meet the needs of frail and disabled communitydwelling older people. International Journal of Integrated Care; 13(May): 17.
9. Elliott, M., Gil, S., Hemmelgarn, B., Manns, B., Tonelli, M., Jun, M., \& Donald, M., (2017): A scoping review of adult chronic kidney disease clinical pathways for primary care. Nephrology Dialysis Transplantation, 32(5), 838-846.

10. Garcı, L., Martı, J. G., Torralba-martı, A., Miguel-perello, J., \& Aguayo-albasini, L., (2014): Complications and level of satisfaction after dermolipectomy and abdominoplasty. Cirugia Espanola journal, 92(4), 254-260.

11. Graeber, S., Richter, S., Folz, J., Jacob, P., \& Schilling, M., (2007). Clinical Pathways in General Surgery.Methods Information In Medicine 46(5):574-579.

12. Hussain, Z., Lim, M., \& Stojkovic, S., (2017): Role of clinical pathway in improving the quality of care for patients with faecal incontinence: A randomised trial. World Journal Gastrointestinal and Pharmacological Therapetutics; 8(1), 81-89.

13. Husted, H., Holm, G., \& Jacobsen, S., (2008): Predictors of length of stay and patient satisfaction after hip and knee replacement surgery: Fast-track experience in 712 patients. Acta Orthopaedica, 79(2), 168-173.

14. Iftikhar, A., Allah, N., Shadiullah, K., Habibullah, K., Muhammad, A., \& Muhammad, H., (2011): Predictors of patient satisfaction.Gomal Journal of Medical Sciences, 9(2), 183-188.

15. Kash, B., \& Mckahan, M., (2017): The Evolution of Measuring Patient Satisfaction. Journal of Primary Health Care and General Practice, 1(1), 2015-2018.

16. Kaye, D., Richardson, C., Ye, Z., Herrel, L., Ellimoottil, C., \& Miller, D., (2017): Association between patient satisfaction and short-term outcomes after major cancer surgery. Annals of Surgical Oncology, 24(12), 34863493.

17. Khanzada T., Samad, A., Memon, W., \& Kumar, B., (2010): Post thyroidectomy complications: the Hyderabad experience. Journal of Ayub Medical College, Abbottabad : JAMC; 22(1): 65-8.

18. Mohamed, M., El-deen, S., Ali, G., \& Ibrahim, M., (2019): Effect of applying a clinical pathway for patients with Congestive Heart Failure on their health status outcomes. Nursing and Palliative Care International Journal; 2(1), 12-19.

19. Rama, M., \& Kanagaluru, S., (2011): A study on the satisfaction of patients with reference to hospital services. International Journal of 
Business Economics \& Management Research, 1, (3).

20. Ramanujam, L., \& Cheah, W., (2005): Improvements in health care for patients undergoing thyroidectomy. Asian journal of surgery / Asian Surgical Association; 28(4): 266-70.

21. Soria, V., Flores, B., Candel, M., Carrillo, A., Campillo, A., Miguel, J., \& Aguayo, J., (2008): Evaluation and monitoring of the clinical pathway for thyroidectomy. The American surgeon Journal; 74(1): 29-36.

22. Yang, Y., Hu, X., Zhang, Q., Cao, H., Li, J., Wang, J., \& Xin, S., (2014): Effect of clinical nursing pathway for endoscopic thyroidectomy in Chinese patients: A meta-analysis. International Journal of Nursing Practice;22(3): 224-231. 